\title{
THE
}

$5-1998$

\section{Hydrodynamic Limits for the Monomer-Dimer Surface Reaction: Chemical Diffusion, Wave Propagation, and Equistability}

\author{
M. Tammaro \\ University of Rhode Island, tammaro@uri.edu
}

J.W. Evans

Follow this and additional works at: https://digitalcommons.uri.edu/phys_facpubs

Terms of Use

All rights reserved under copyright.

\section{Citation/Publisher Attribution}

Tammaro, M., \& Evans, J. W. (1998). Hydrodynamic limits for the monomer-dimer surface reaction: Chemical diffusion, wave propagation, and equistability. Physical Review E, 57(5), 5087-5094. doi: 10.1103/PhysRevE.57.5087

Available at: http://dx.doi.org/10.1103/PhysRevE.57.5087

This Article is brought to you for free and open access by the Physics at DigitalCommons@URI. It has been accepted for inclusion in Physics Faculty Publications by an authorized administrator of DigitalCommons@URI. For more information, please contact digitalcommons-group@uri.edu. 


\title{
Hydrodynamic limits for the monomer-dimer surface reaction: Chemical diffusion, wave propagation, and equistability
}

\author{
M. Tammaro \\ Department of Physics, University of Rhode Island, Kingston, Rhode Island 02881 \\ J. W. Evans \\ Ames Laboratory and Department of Mathematics, Iowa State University, Ames, Iowa 50011
}

(Received 18 December 1997)

\begin{abstract}
For finite adspecies mobility, the lattice-gas monomer-dimer $\left(A+B_{2}\right)$ surface reaction model exhibits a discontinuous transition from a stable reactive steady state to a stable $A$-poisoned steady state, as the impingement rate $P_{A}$ for $A$ increases above a critical value $P^{*}$. The reactive (poisoned) state is metastable for $P_{A}$ just above (below) $P^{*}$. Increasing the surface mobility of $A$ enhances metastability, leading to bistability in the limit of high mobility. In the bistable region, the more stable state displaces the less stable one separated from it by a planar interface, with $P^{*}$ becoming the equistability point for the two states. This hydrodynamic regime can be described by reaction-diffusion equations (RDE's). However, for finite reaction rates, mixed adlayers of $A$ and $B$ are formed, resulting in a coverage-dependent and tensorial nature to chemical diffusion (even in the absence of interactions beyond site blocking). For equal mobility of adsorbed $A$ and $B$, and finite reaction rate, the prediction for $P^{*}$ from such RDE's, incorporating the appropriate description of chemical diffusion, is shown to coincide with that from kinetic Monte Carlo simulations for the lattice-gas model in the regime of high mobility. Behavior for this special case is compared with that for various other prescriptions of mobility, for both finite and infinite reaction rates. [S1063-651X(98)07905-7]
\end{abstract}

PACS number(s): 05.60.+w, 05.40. $+\mathrm{j}, 82.65 . J v$

\section{INTRODUCTION}

In most surface reactions on single crystal substrates, adlayer ordering and mixing significantly influence both the reaction kinetics and the chemical diffusion of adspecies across the surface. Atomistic lattice-gas (LG) treatments provide the possibility to incorporate and accurately describe such effects [1], which are ignored in traditional mean-field (MF) treatments [2]. However, most LG modeling has failed to recognize and incorporate the feature that rates for hopping between surface sites, for at least some adsorbed reactants, are typically many orders of magnitude larger than rates for all other processes (adsorption, reaction, and desorption) [3]. Indeed, this rapid mobility is responsible [4-7] for strong hysteresis and metastability in the reaction kinetics, and for the mesoscopic length scales of spatial pattern formation, observed in these systems [2]. Thus it is important to focus on the behavior of LG models in the appropriate "hydrodynamic limit" of rapid mobility of these reactants [4-7].

There have been numerous studies of the classic monomer-dimer $\left(A+B_{2}\right)$ surface reaction model, which thus provides a natural testing ground for the above issues. Early MF studies revealed bistability between a reactive steady state, with low monomer coverage, and a monomer-poisoned steady state [8]. MF reaction-diffusion equation studies of the evolution of a chemical wave separating these states allows assessment of their relative stability [4]. The initial LG model studies focused on the case without surface mobility of reactants, where "large" fluctuations readily produce a discontinuous transition between the reactive and poisoned states $[9,10]$. Subsequent studies introduced limited mobility to examine its effect on the location of the transition $[11,12]$.
There have even been a few more comprehensive studies examining behavior of both the reaction kinetics and steady states (including the enhancement of metastability and hysteresis), as well as the characteristics of chemical wave propagation, in the regime of high mobility $[4-7,13]$. However, there has been no previous study for finite reaction rate of the canonical case of equal mobility of monomers and dimers (which can hop to adjacent empty sites), or any systematic examination of how the prescription of mobility affects behavior in the hydrodynamic limit. These issues are addressed in this contribution.

In Sec. II we describe the LG monomer-dimer surface reaction model, and the basic properties of the model. A reaction-diffusion equation (RDE) formalism to describe behavior in the hydrodynamic limit of large monomer mobility is presented in Sec. III. In Sec. IV we discuss appropriate descriptions of chemical diffusion in mixed adlayers. These descriptions are incorporated into the RDE's. An analysis of behavior of the LG model with equal mobility of monomers and dimers, and with a reaction rate of unity, is presented in Sec. V. Behavior in the limit of high mobility is compared with predictions of the appropriate RDE's. A comprehensive listing and comparison of values for the equistability point, $P_{A}=P^{*}$, is given in Sec. VI for various prescriptions of mobility, for both finite and infinite reaction rates. Some concluding remarks are presented in Sec. VII.

\section{LATTICE-GAS MONOMER-DIMER $\left(A+B_{2}\right)$ SURFACE REACTION MODEL}

The monomer-dimer $\left(A+B_{2}\right)$ lattice-gas reaction model includes the steps $[8-10]$ 


$$
\begin{gathered}
A(\text { gas })+E \stackrel{P_{A}}{\longrightarrow} A(\text { ads }), \\
B_{2}(\text { gas })+2 E \stackrel{P_{B}}{\longrightarrow} 2 B(\text { ads }), \\
A(\text { ads })+B(\text { ads }) \stackrel{k}{\longrightarrow} A B(\text { gas })+2 E,
\end{gathered}
$$

where "gas" denotes gas phase, "ads" denotes adsorbed species, and $E$ denotes an empty surface adsorption site. To describe the model more explicitly: $A$ (gas) adsorbs at rate $P_{A}$ on single empty sites; $B_{2}$ (gas) adsorbs at rate $P_{B}$ on adjacent pairs of empty sites; and adjacent $A$ (ads) and $B$ (ads) react to form the product $A B$ (gas) at rate $k$ for each $A$ (ads)- $B$ (ads) pair. We also allow hopping of $A$ (ads) and $B$ (ads) to any adjacent empty site with rates $h_{A}$ and $h_{B}$, respectively, and "exchange diffusion" of each adjacent $A$ (ads) and $B$ (ads) at rate $h_{A B}$. We assume here that $h_{A}$ $\geqslant h_{B}$ and $h_{A} \geqslant h_{A B}$. These diffusion processes may be represented schematically as

$$
\begin{gathered}
A(\text { ads })+E \stackrel{h_{A}}{\longrightarrow} E+A(\text { ads }), \\
B(\text { ads })+E \stackrel{h_{B}}{\longrightarrow} B(\text { ads })+E \\
A(\text { ads })+B(\text { ads }) \stackrel{h_{A B}}{\longrightarrow} B(\text { ads })+A(\text { ads }) .
\end{gathered}
$$

Thus, implicitly, in this model, there are no interactions between adspecies, other than exclusion of double site occupancy, and reaction of adjacent $A$ (ads) and $B$ (ads). Below, we choose $P_{A}+P_{B}=1$, which sets the time scale. We shall consider finite reaction rates (with $k=1$ ), and also instantaneous reaction, where $k \rightarrow \infty$. This model has the same Langmuir-Hinshelwood adsorption and reaction steps as $\mathrm{CO}$ oxidation [2], if one makes the identifications $A \leftrightarrow \mathrm{CO}$ and $B_{2} \leftrightarrow \mathrm{O}_{2}$. Below, coverages (in monolayers, ML) for $A$ (ads) and $B$ (ads) will be denoted by $\theta_{A}$ and $\theta_{B}$, respectively, and the total coverage by $\theta=\theta_{A}+\theta_{B}$.

For finite hop rates, the surface reaction model exhibits a discontinuous transition from a stable reactive steady state with low $\theta_{A}$, for some or all $P_{A}<P^{*}$, to a stable $A$-poisoned steady state with $\theta_{A}=1$, for $P_{A}>P^{*}[5,7,9-13]$. The location of the transition, $P_{A}=P^{*}$, is nontrivial. A metastable reactive state persists for some finite range $P^{*}$ $<P_{A}<P^{s+}$ bounded above by an upper spinodal point $P^{s+}$ (the precise definition of which is unclear) $[6,14]$. Of course, the $A$-poisoned steady state is an absorbing state, which exists (with infinite lifetime) for all $P_{A}$. However, it is only stable, attracting nonpoisoned states, for $P_{A}>P^{*}$. It appears to be metastable (only transiently attracting nearly $A$-poisoned states) for some range $P^{s-}<P_{A}<P^{*}$, and to be unstable for lower $P_{A}$ (repelling nonpoisoned states) [6]. Here $P^{s-}$ denotes a lower spinodal. [For completeness, we note that there is also a $B$-poisoned adsorbing steady state, which is typically unstable. The only exception is for limited mobility of the dimer species, and for a range of $P_{A}<P^{* *}$ (with $P^{* *}<P^{*}$ ), where it becomes stable. In this case, there is a continuous transition to the stable reactive state at $P_{A}$ $=P^{* *}$. Although this transition has been the focus of numerous studies [1], it does not significantly impact upon the issues considered here, and is thus deemphasized.]
With increasing mobility of $A$ (ads), the lifetime of the metastable reactive state increases and eventually diverges, and the width of its existence range $P^{s+}-P^{*}$ expands significantly. The width of the existence range $P^{*}-P^{s-}$ of the metastable $A$-poisoned state, also expands to $P^{*}$, since $P^{s-} \rightarrow 0$. In the hydrodynamic regime,', where $h_{A} \rightarrow \infty$, fluctuations in nonpoisoned states are quenched, true bistability is achieved, and $P^{*}$ becomes the equistability point for the bistable reactive and $A$-poisoned states [4-7].

Of particular relevance for this study is the observation that for $P_{A}<P^{*}$, a stable reactive state will displace the metastable or unstable $A$-poisoned state, separated from it by an on-average planer interface. This creates a trigger or chemical wave of velocity $V>0$, say. For $P^{*}<P_{A}<P^{s+}$, the stable $A$-poisoned state displaces metastable reactive state, creating a trigger wave with velocity $V<0$ (at least until the metastable reactive state spontaneously poisons). This velocity, $V$, vanishes as $P_{A} \rightarrow P^{*}$, where the reactive and $A$-poisoned states are in equistable coexistence. See Refs. [5-7,13]. Near the transition, one has $V \approx k_{c}\left(L_{r}^{2}\right.$ $\left.+L_{d}^{2}\right)^{1 / 2}\left(P^{*}-P_{A}\right)$, where $k_{c}=O(1)$ is the overall characteristic rate for the reaction, $L_{r}=O(1)$ is the range of direct spatial contact due to nearest-neighbor reaction and dimer adsorption mechanisms, and $L_{d} \sim\left(h_{A} / k_{c}\right)^{1 / 2}$ is the diffusion length (for $h_{A} \geqslant h_{B}$ and $h_{A B}$ ) [5,7]. Here, distances are measured in units of the surface lattice constant $a$.

\section{HYDRODYNAMIC REGIME OF THE $A+B_{2}$ MODEL}

In the hydrodynamic regime, where $h_{A}$ and possibly also other hop rates are very large, the local coverages, $\theta_{A}$ and $\theta_{B}$, can vary not just in time, but also spatially on a macroscopic length scale controlled by the diffusivity. This spatiotemporal variation is described by reaction-diffusion equations of the form [7]

$$
\begin{gathered}
(\partial / \partial t) \theta_{A}=P_{A} \theta_{E}-z k \theta_{A B}-\underline{\nabla} \cdot \underline{J}_{A} \quad \text { and } \\
(\partial / \partial t) \theta_{B}=2 P_{B} \theta_{E E}-z k \theta_{A B}-\underline{\nabla} \cdot \underline{J}_{B} .
\end{gathered}
$$

Here $z$ is the coordination number of the lattice, $\theta_{A B}$ is the local probability that any adjacent pair of sites has one specified site occupied by $A$, and the other by $B$ (i.e., $\theta_{A B}$ is the "coverage" of $A B$ pairs), and $\theta_{E E}$ is the local probability that sites in an adjacent pair are both empty (i.e., $\theta_{E E}$ is the "coverage", of $E E$ pairs). For a completely randomized adlayer, one has $\theta_{A B}=\theta_{A} \theta_{B}$, and $\theta_{E E}=\left(\theta_{E}\right)^{2}$. However, in the presence of spatial correlations as is the case of finite mobility of $B$ (ads), these simple relations are not satisfied, and Eq. (1) should be regarded as the lowest order equations in an infinite hierarchy [6]. In Eq. (1), $\underline{J}_{K}$ denotes the diffusive flux of adspecies $K=A$ or $B$. In general, a gradient in the coverage of one adspecies generates a diffusive flux in both adspecies, so one writes [15]

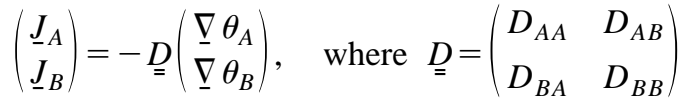

is a tensor of diffusion coefficients. See Sec. IV for further discussion. 

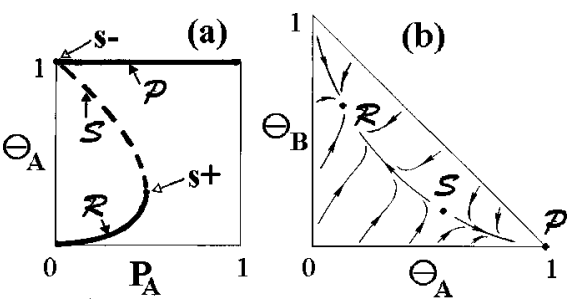

FIG. 1. (a) Schematic of steady-state behavior of $\theta_{A}$ (in ML) versus $P_{A}$ in the hydrodynamic limit, and in the absence of a $B$-poisoned state. $\mathcal{R}, \mathcal{P}$, and $\mathcal{S}$ denote the reactive, $A$-poisoned, and unstable states, respectively; $s+(s-)$ denotes the upper (lower) spinodal. (b) Schematic of the evolution of $\theta_{A}$ and $\theta_{B}$ (in ML) for a spatially homogeneous system in the bistable regime.

First, we discuss the steady-state behavior and kinetics for spatially homogeneous systems $[4,6,8]$. A stable $A$-poisoned steady state $(\mathcal{P})$ exists for all $P_{A}$. In a bistable regime with $0=P^{s-} \leqslant P_{A} \leqslant P^{s+}$, there also exists a stable reactive steady state $(\mathcal{R})$ [except for limited mobility of $B($ ads), where this state is replaced by a stable $B$-poisoned state for $\left.P_{A}<P^{* *}\right]$. In the bistable regime, there also exists an unstable steady state or "saddle point" $(\mathcal{S})$, using the terminology of nonlinear dynamics, which "connects" the stable states. The upper (lower) spinodal $s+(s-)$ located at $P_{A}=P^{s+}\left(P_{A}\right.$ $\left.=P^{s-}=0\right)$, which bounds the bistable regime, corresponds to a saddle-node (transcritical) bifurcation. Figure 1(a) provides a schematic of the steady-state behavior for $\theta_{A}$, and Fig. 1(b) indicates schematically the dynamics of the homogeneous system in the bistable regime. If $B$ (ads), as well as $A$ (ads), is highly mobile, the adlayer is randomized, and both stable and unstable steady-state behavior is exactly determined from MF rate equations. For limited mobility of $B$ (ads), nontrivial spatial correlations persist, but one can precisely analyze the behavior of the stable reactive state by conventional simulation techniques, and that of the unstable state by unconventional constant-coverage ensemble simulation techniques [6].

Next, we discuss chemical wave propagation in the regime of bistability for this model. Specifically, we consider the propagation in the $x$ direction, with velocity $V$, of planar waves which separate the $A$-poisoned state on the left, from the reactive state on the right. Such waves correspond to solutions of the RDE's (1) of the form $\theta_{A}=\theta_{A}(\xi=x-V t)$, $\theta_{B}=\theta_{B}(\xi=x-V t), \theta_{A B}=\theta_{A B}(\xi=x-V t)$, etc. Here, it is useful to introduce a quasimechanical terminology (cf. Ref. [16]): $X=\left(\theta_{A}, \theta_{B}\right)^{T}$ denotes the "position" of a quasiparticle at "time" $\xi ; D$ denotes its position-dependent "tensor mass"; $\underline{P}=\underline{D}(\partial / \partial \xi) \underline{X}$ denotes its "momentum"; and $\underline{F}=$ $\left(-P_{A} \theta_{E}+z k \theta_{A B},-2 P_{B} \theta_{E E}+z k \theta_{A B}\right)^{T} \quad$ denotes the position-dependent "force" field in which the quasiparticle moves [17]. Then, the poisoned state $(\mathcal{P})$ and reactive steady state $(\mathcal{R})$ correspond to unstable equilibrium points in the force field, and the unstable state $(\mathcal{S}$ ) corresponds to a equilibrium saddle point [cf. Fig. 1(b), where the arrows point in the opposite direction of the force vector $F]$. Then, the RDE's can be recast in the form of Newton's equations, including an extra drag (antidrag) force term, for $V>0$ ( $V$ $<0)$. Specifically, one has

$$
(\partial / \partial \xi) \underline{P}=\underline{F}-V(\partial / \partial \xi) \underline{X} .
$$

The profile of the chemical wave corresponds to a specially selected trajectory of the quasiparticle which starts almost at rest at $\mathcal{P}$, and ends up at rest at $\mathcal{R}$. If $\mathcal{P}$ is more (less) stable than $\mathcal{R}$ in the RDE's, it corresponds to the less (more) stable equilibrium point of $\underline{F}$, in that a drag (antidrag) force, $V>0(V<0)$, is required to achieve the above motion. In the case of equistability, which is of primary interest below, the particle achieves this motion between the unstable equilibrium points without any drag force $(V=0)$. We note that when $D$ is diagonal with $D_{A A}=D_{B B}$, and in some other cases for instantaneous reaction, Eq. (3) becomes a conventional Newton's equation (see Sec. VI).

Finally, we briefly comment on the limiting case, $k \rightarrow \infty$. Such instantaneous reaction, together with rapid mobility in the hydrodynamic regime, means that only one type of adspecies can populate any macroscopic point in space $[4,5]$. In regions populated by $A$ (ads), the adlayer is completely randomized, since $h_{A} \rightarrow \infty$. Here, the reaction rate must approach the $B$-adsorption rate, so $z k \theta_{A B} \rightarrow 2 P_{B} \theta_{E E}$ $=2 P_{B}\left(\theta_{E}\right)^{2}$, and one can set $\theta_{E}=1-\theta_{A}$ in both the adsorption and reaction terms of Eq. (1) [4]. In regions populated by $B$ (ads), the reaction rate approaches the $A$-adsorption rate, so $z k \theta_{A B} \rightarrow 2 P_{A} \theta_{E}$, but the adlayer is only completely randomized if $h_{B} \rightarrow \infty$, which somewhat complicates the analysis [4].

\section{CHEMICAL DIFFUSION IN MIXED ADLAYERS OF $A$ (ads) AND $B$ (ads)}

Again we consider the hydrodynamic regime, where $h_{A}$ and possibly also other hop rates are very large. For a reaction rate which is comparable to or lower than the total adsorption rate (and thus for $k=1$ ), the steady states in the reaction model are mixed adlayers with significant local coverages of both $A$ (ads) and $B$ (ads). This feature implies that the description of chemical diffusion is nontrivial, even in the absence of adspecies interactions (beyond site blocking). One can, however, enumerate some properties of $D$ for general hop rates. Below, we let $D_{K}^{0}=z a^{2} h_{K} / 4$ denote the singleparticle diffusion coefficients, for $K=A$ and $B$ (where $a$ is the surface lattice constant). Note that, due to the lack of adspecies interactions, $D_{K}^{0}$ also corresponds to the chemical diffusion coefficient for an adlayer populated by a single species, $K$ [18]. For general mixed adlayers (in the absence of interactions beyond site blocking), one has [15] the following features.

(a) $(\underline{D})_{J K}=D_{J K} \rightarrow \delta_{J K} D_{K}^{0}$, as both $\theta_{A} \rightarrow 0$ and $\theta_{B} \rightarrow 0$, since there is negligible interference of surface diffusion by coadsorbed species.

(b) $D_{A B} \rightarrow 0$, as $\theta_{A} \rightarrow 0$, and $D_{B A} \rightarrow 0$, as $\theta_{B} \rightarrow 0$, since the diffusive flux in adspecies $K$ induced by a gradient in the coadsorbed species must vanish with the coverage of $K$.

(c) If $h_{A B}$ is negligible compared with the diverging hop rate $h_{A}$, then $\underline{J}_{K} \rightarrow \underline{0}$, as $\theta_{A}+\theta_{B} \rightarrow 1$, since the lack of vacancies on the "jammed" surface precludes significant diffusion. When $\theta_{A}+\theta_{B}=1$, one has $\nabla \theta_{A}=-\nabla \theta_{B}$, so $\underline{J}_{K}=\underline{0}$ implies that $D_{K A}=D_{K B}$.

We now give the explicit form of the diffusion coefficients in some special cases. For all these, the diverging hop rate(s) will be denoted by $h$, and we set $D^{0}=z a^{2} h / 4$. See the Appendix for further discussion of these and other cases. 
(i) $h_{A}=h_{B}=h_{A B}=h \rightarrow \infty$. Here, each type of adspecies diffuses independent of the other. Furthermore, due to the lack of interactions, chemical diffusion is described by single-particle equations [18]. Thus one has "simple diffusion" described by $D_{J K}=\delta_{J K} D^{0}$.

(ii) $h_{A}=h_{B}=h \rightarrow \infty ; h_{A B} / h \rightarrow 0$. Here, one can exploit special physical and symmetry properties to analyze $D \underline{D}[15]$. The total coverage, $\theta=\theta_{A}+\theta_{B}$, must satisfy the classic diffusion equation for a noninteracting single-species lattice gas with hop rate $h$. Thus it follows that $D_{A A}+D_{B A}$ $=D_{A B}+D_{B B}=D^{0}$. Furthermore, if $D_{A A}=D_{\theta_{A}}\left(\theta_{B}\right)$, then by symmetry $D_{B B}=D_{\theta_{B}}\left(\theta_{A}\right)$, so $\underset{D}{D}$ is determined by the single function $D_{y}(x)$. In the limit $y \rightarrow 0+, D_{0+}(x)=D_{\mathrm{tr}}(x)$ $=D^{0}(1-x) f(x)$ reduces to the conventional tracer diffusion coefficient for a particle in a bath of particles of density $x$ with the same hop rate. The "correlation factor" $f(x) \leqslant 1$ reflects a tendency for backward hopping of the tracer particle. Simulations show that $f(x) \approx 1-0.62 x+0.08 x^{2}$ for random adlayers. Since $D_{A B}+D_{B B}=D^{0}$, and $D_{B A}$ $=D_{A A}$ at jamming, where $y \rightarrow 1-x$, one also has $D_{1-x}(x)$ $+D_{x}(1-x)=D^{0}$, indicating that $D_{1-x}(x)=D^{0}(1-x)$. More generally, one can show that $[15,19]$

$$
\begin{gathered}
D_{y}(x)=p_{y} D^{0}+p_{x} D_{\operatorname{tr}}(y+x) \text { for } 0 \leqslant y \leqslant 1-x, \\
\text { where } p_{x}=x /(x+y) \text { and } p_{y}=y /(x+y) .
\end{gathered}
$$

(iii) $h_{A}=h_{A B}=h \rightarrow \infty ; h_{B} / h \rightarrow 0$. In this case, the diffusion of $A$ (ads) is independent of $B$ (ads), so $D_{A A}=D^{0}$ and $D_{A B}=0$. It is also clear that diffusion of each $B$ (ads) is independent of other $B(\mathrm{ads})$, so one expects that $D_{B B}=\theta_{A} D^{0}$ and $D_{B A}=-\theta_{B} D^{0}$.

(iv) $h_{A}=h \rightarrow \infty ; h_{B} / h$ and $h_{A B} / h \rightarrow 0$. Here $A$ (ads) diffuses rapidly through an effectively immobile background of coadsorbed $B($ ads $)$. Due to the lack of interactions, $D_{A A}$ corresponds to the "percolative" diffusion coefficient for a single particle of $A$ (ads). $D_{A A}=D_{\text {perc }}\left(\theta_{B}\right)$ decreases from $D^{0}$ to zero, as $\theta_{B}$ increases from zero to a percolation threshold, where paths of sites not occupied by $B$ (ads) cease to span the system. $D_{\text {perc }}$ actually depends on the full configuration $\{B\}$ of $B$ (ads), since spatial correlations in this distribution affect both the percolation and transport properties [7]. Also, due to the lack of interactions, the diffusive flux of $A$ (ads) induced by a gradient in $\theta_{B}$ is directly proportional to $\theta_{A}$, i.e., $D_{A B}=\theta_{A} G\left(\theta_{B}\right)$. The equality $D_{A A}=D_{A B}$, when $\theta$ $=\theta_{A}+\theta_{B}=1$, then implies that $G\left(\theta_{B}\right)=D_{\text {perc }}\left(\theta_{B}\right) /\left(1-\theta_{B}\right)$ [7]. Clearly, here one has $D_{B B}=D_{B A}=0$.

The case of instantaneous reaction deserves special comment. As noted in Sec. III, here only one adspecies can occupy a macroscopic point (so only one of $\theta_{A}$ and $\theta_{B}$ can be nonzero at a single point). Thus the diffusion in such a single-species region of the adlayer is trivial (in the absence of adspecies interactions), being described by a constant single-particle diffusion coefficient $[4,5,18]$.

\section{DETAILED ANALYSIS OF THE $A+B_{2}$ MODEL FOR EQUAL MOBILITY OF $A($ ads) AND $B($ ads) AND FINITE REACTION RATE $(k=1)$}

Here, we first present results from a simulation study of the $A+B_{2}$ model with $h_{A}=h_{B}=h, h_{A B}=0$, and $k=1$. Simu-

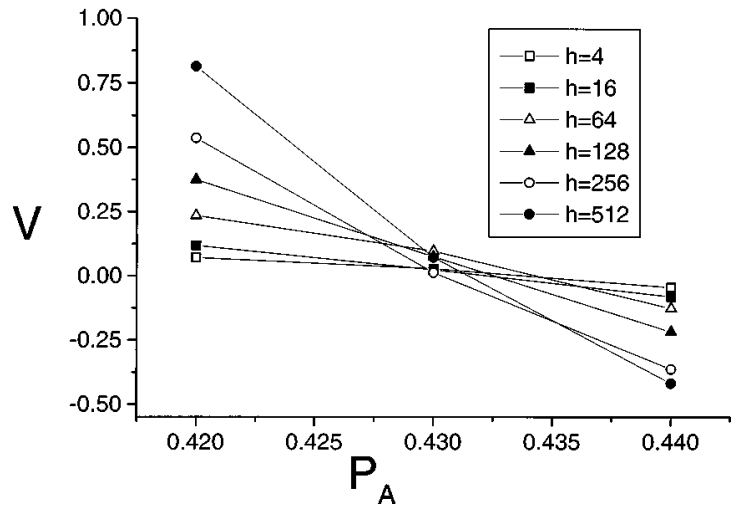

FIG. 2. Propagation velocity $V$ (measured in lattice constants per unit time) versus $P_{A}$, for an on-average planar interface separating $A$-poisoned and reactive states. Behavior is only shown for a narrow range of $P_{A}$ close to $P^{*}$ (where $V=0$ ). Here $k=1, h_{A B}$ $=0$, and behavior is shown for various values of $h_{A}=h_{B}=h$ (indicated).

lations were performed on a $600 \times 1000$ site square lattice with periodic boundary conditions. We focus on the propagation of a planar interface between the reactive and $A$-poisoned states. Specifically, a band of the $A$-poisoned state is placed across an otherwise empty lattice (aligned the short side). After the reaction process is initiated for some $P_{A}<P^{s+}$, a stable or metastable reactive state is "quickly" formed on the empty region of the lattice, and then a chemical wave develops associated with the contraction (for $P_{A}$ $<P^{*}$ ) or expansion (for $P_{A}>P^{*}$ ) of the $A$-poisoned band immersed in the reactive state.

From analysis of such evolution, we obtain the variation with $P_{A}$ of the interface propagation velocity $V$, measured in surface lattice constants per unit time. This behavior is shown in Fig. 2, for various $h$ values indicated. We consider only a narrow range of $P_{A}$ around the location of the discontinuous transition, $P_{A}=P^{*}$, where $V=0$ [noting that $P^{*}$ $=P^{*}(h)$ varies little with $\left.h\right]$. As expected (cf. Sec. II), the slope of these curves increases with $h_{A}$. However, there is also a "near-crossing feature" in that the curves tend to roughly pivot about a single point $P_{A} \approx P_{x}=0.430 \pm 0.003$ and $V=0.05 \pm 0.05$. Analogous behavior has been observed previously for other cases of the monomer-dimer surface reaction model $[7,13]$. This feature is particularly useful as it allows ready extrapolation to assess $P^{*}(h \rightarrow \infty)$ [7]. When $h \rightarrow \infty, V$ versus $P_{A}$ must be represented by a vertical line on the scale of this plot, located at $P_{A}=P^{*}(h \rightarrow \infty) \approx P_{x}$ $\approx 0.430$. Thus $P_{x}=0.430 \pm 0.003$ provides an estimate of the location of the equistability point in the hydrodynamic limit. Finally, in Fig. 3, we show predictions from simulations with large $h=512$ for the variation of the scaled velocity, $\nu$ $=V / h^{1 / 2}$, over a broader range of $P_{A}$. This scaled velocity for $h=512$ has effectively converged to the finite value corresponding to the hydrodynamic limit $h \rightarrow \infty$.

Next, we compare these predictions from the simulations for large- $h$ hydrodynamic behavior with those of appropriate reaction-diffusion equations of the form of Eq. (1). First, we note that since both $A(\mathrm{ads})$ and $B(\mathrm{ads})$ are highly mobile on the time scale of adsorption and reaction, there will be no spatial correlations in the adlayer. As a result, a simple mean-field treatment of adsorption and reaction kinetics can 


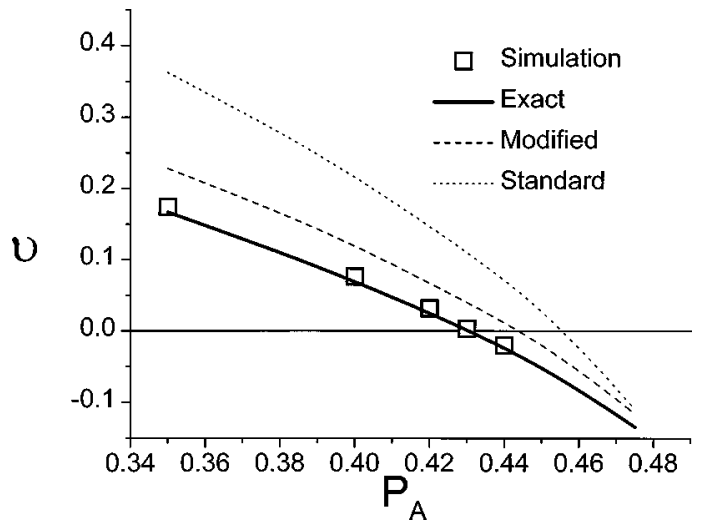

FIG. 3. Scaled propagation velocity $\nu=V / h^{1 / 2}$ versus a broad range of $P_{A}$, for an on-average planar interface separating $A$-poisoned and reactive states, in the $A+B_{2}$ model for $k=1$. Simulation results (symbols) are for $h_{A B}=0$, and $h_{A}=h_{B}=h=512$. RDE results (curves) are for mean-field kinetics, and various prescriptions of diffusion described in the text.

be used in the RDE's and, in particular, one finds that $P^{s+}$ $=\frac{1}{2}$. In contrast, the description of chemical diffusion for this choice of equal adspecies mobility is nontrivial. However, the appropriate exact prescription is provided by case (ii) in Sec. IV. The predictions of such exact RDE's for the scaled velocity $\nu$ versus $P_{A}$, are shown in Fig. 3, and are completely consistent with predictions from the simulation behavior for large $h$. In particular, one obtains a precise value of $P^{*}=0.430$ from these RDE's.

For contrast, in Fig. 3, we have also shown the predictions for $\nu$ versus $P_{A}$ of reaction-diffusion equations with exact mean-field treatments of kinetics, but with approximate treatments of diffusion. The "standard" treatment of diffusion, where $(\underline{D})_{J, K}=D^{0} \delta_{J, K}$, ignores the interference of coadsorbed species on diffusion. This substantially overestimates $\nu$ for $P_{A}<P^{*}$, and predicts that $P^{*}=0.456$. In a "modified" treatment of diffusion, which accounts in an approximate way (and actually underestimates) the influence of coadsorbed species on diffusion $[6,7,15]$, we set $(\underline{\underline{D}})_{J, K}$ $=D^{0}\left(1-\theta_{J^{\prime}}\right) \delta_{J, K}+D^{0} \theta_{J}\left(1-\delta_{J, K}\right)$, where $J^{\prime}=A(B)$ when $J=B(A)$. As one might expect, the corresponding predictions for $\nu$ versus $P_{A}$, and thus for $P^{*}=0.444$, are considerably closer to the exact values.

Finally, as well as considering the propagation velocity and equistability point, one can also examine the coverage profiles across the chemical wave front. Here, we consider these profiles only for $P_{A}=P^{*}$. In Fig. 4, we compare such profiles obtained from simulations for large $h=128$ with those from reaction-diffusion equations with various prescriptions of chemical diffusion. [In the simulations on a square lattice with sites labeled by $(i, j)$, we have simply determined the average concentration of both adspecies along rows, $j$, orthogonal to the direction of propagation, $i$.] As expected, the profile shape is reproduced by RDE's with the exact treatment of diffusion, but not by those with the approximate "standard" and "modified" treatments. For purposes of comparison between the simulations and RDE's, we have shown coverages as a function of the appropriately scaled position, $i / h^{1 / 2}$ or $x /\left(D^{0}\right)^{1 / 2}$.

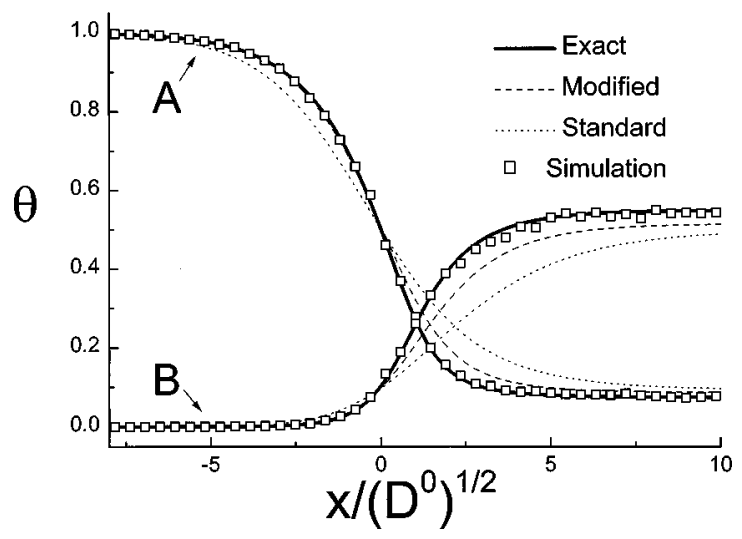

FIG. 4. Coverage profiles for the stationary interface between $A$-poisoned and reactive states in the $A+B_{2}$ model with $k=1$ at equistability. Spatial coordinates are rescaled. Simulation results (symbols) are for $h_{A B}=0$, and $h_{A}=h_{B}=h=128$. RDE results (curves) are for mean-field kinetics, and various prescriptions of diffusion described in the text.

\section{BEHAVIOR FOR OTHER CHOICES OF MOBILITY AND REACTION RATE}

It is instructive to compare $A+B_{2}$ reaction model behavior for the choice of parameters considered in Sec. V with that obtained for different prescriptions of adspecies mobility (retaining a reaction rate of $k=1$ ), and with that for instantaneous reaction, $k=\infty$. Here, we focus on results for the equistability point, $P_{A}=P^{*}$, which are summarized in Table I.

We first elaborate on these results for the case of finite reaction rate, $k=1$.

(i) $h_{A}=h_{B}=h_{A B}=h \rightarrow \infty$. As noted in Sec. IV, and in the Appendix, the traditional description of diffusion applies here, i.e., $D_{J K}=\delta_{J K} D^{0}$. Furthermore, the rapid diffusion of both adspecies guarantees that the adlayer is randomized, so the mean-field description of kinetics applies, and $P^{s+}=\frac{1}{2}$. Analysis of the appropriate traditional RDE's then yields [4] $P^{*}=0.456$.

(ii) $h_{A}=h_{B}=h \rightarrow \infty$ and $h_{A B} / h \rightarrow 0$. This is the case analyzed in Sec. IV, where mean-field kinetics applies (so again $P^{s+}=\frac{1}{2}$ ), but where diffusion is nontrivial due to the influence of coadsorbed species. We found that $P^{*}=0.430$.

TABLE I. Location of the equistability point $P_{A}=P^{*}$ in the hydrodynamic limit of the $A+B_{2}$ reaction model with various prescriptions of adspecies mobility. Results are shown for both finite reaction rate, $k=1$ (where $P^{s+} \approx \frac{1}{2}$ ), and instantaneous reaction, $k$ $=\infty \quad\left(\right.$ where $\left.P^{s+}=\frac{2}{3}\right)$. For $k=\infty$, exact $P^{*}$ values are (a) $(2 \sqrt{3}-2) /(2 \sqrt{3}-1)$ and (b) $\frac{4}{7}$.

\begin{tabular}{lcc}
\hline \hline & $k=1$ & $k=\infty$ \\
\hline$h_{A}=h_{B}=h_{A B}=h \rightarrow \infty$ & 0.456 & $0.594(\mathrm{a})$ \\
$h_{A}=h_{B}=h \rightarrow \infty$ and $h_{A B} / h \rightarrow 0$ & 0.430 & $0.594(\mathrm{a})$ \\
$h_{A}=h_{A B}=h \rightarrow \infty$ and $h_{B} / h \rightarrow 0$ & 0.450 & $0.571(\mathrm{~b})$ \\
$h_{A}=h \rightarrow \infty, h_{B} \rightarrow \infty$ with $h_{B} / h \rightarrow 0$ & 0.406 & $0.571(\mathrm{~b})$ \\
$\quad$ and $h_{A B} / h \rightarrow 0$ & & \\
$h_{A}=h \rightarrow \infty, h_{B}=h_{A B}=0$ & 0.397 & $0.571(\mathrm{~b})$ \\
\hline \hline
\end{tabular}


(iii) $h_{A}=h_{A B}=h \rightarrow \infty$, and $h_{B} / h \rightarrow 0$. As noted in Sec. V and the Appendix, here the traditional description of diffusion applies for $A$ (ads), but not for $B$ (ads). This prescription of rapid mobility also ensures a randomized adlayer, and thus mean-field kinetics, and $P^{s+}=\frac{1}{2}$. Analysis of the appropriate RDE's yields $P^{*}=0.450$.

(iv) $h_{A}=h \rightarrow \infty, h_{B} \rightarrow \infty$ with $h_{B} / h \rightarrow 0$, and $h_{A B} / h \rightarrow 0$. Since $h_{B} / h \rightarrow 0, A$ (ads) diffuses through an effectively immobile background of coadsorbed $B$ (ads), so diffusion is percolative. Since also $h_{B} \rightarrow \infty$, the $B$ (ads) distribution is randomized, so "simple" random percolation applies, as well as mean-field kinetics, and $P^{s+}=\frac{1}{2}$. Analysis of the appropriate RDE's yields [20] $P^{*}=0.406$.

(v) $h_{A}=h \rightarrow \infty, h_{B}=h_{A B}=0$. Again, diffusion of $A$ (ads) is percolative. However, now $B$ (ads) is immobile, and spatial correlations develop in the $B$ (ads) distribution which affect the kinetics, and the percolation and transport properties [7] (although we still find that $P^{s+} \approx \frac{1}{2}$ ) [6]. Application of a hybrid simulation procedure [6,7] to account for such spatial correlations at distinct macroscopic points across the chemical wave front, or analysis of appropriate correlated RDE's, yields $P^{*}=0.397$.

Continuous transitions in the value of $P^{*}$ between various of these special cases are possible. For example, let $h_{A} \rightarrow \infty$ and retain finite $h_{B}$, and set $h_{A B}=0$. Then for $h_{B}$ increasing from zero to infinity, $P^{*}$ will increase continuously from 0.397 to 0.406 . On the other hand, if one takes $h_{A} \rightarrow \infty$ and $h_{B} \rightarrow \infty$, with fixed ratio $\alpha=h_{B} / h_{A}$, and sets $h_{A B}=0$, then $P^{*}$ will increase continuously from 0.406 to 0.430 , as $\alpha$ increases from $0+$ to 1 .

Next, we consider the case of instantaneous reaction, $k$ $=\infty$. As noted above, here chemical diffusion in the hydrodynamic limit is artificially simple, since there is no adlayer mixing, i.e., each macroscopic point is only populated by one type of adspecies. (It is also clear that behavior is independent of $h_{A B}$, for finite hop rates, since adjacent $A-B$ pairs react before exchanging.) Analysis of the simplified reaction kinetics for the randomized $A$ (ads)-populated region shows immediately that $P^{s+}=\frac{2}{3}$ (rather than $\frac{1}{2}$ when $k=1$ ) $[4,21]$. Furthermore, it is clear that the chemical wave front consists of a region populated by $A$ (ads), on the left (say), separated from a region populated by $B(\mathrm{ads})$, on the right, at a single macroscopic point where both $\theta_{A}$ and $\theta_{B}$ vanish.

If both $h_{A} \rightarrow \infty$ and $h_{B} \rightarrow \infty$, the adlayers are randomized, and the $A$ (ads)- and $B$ (ads)-populated regions are respectively described by the RDE's (cf. Sec. II) [4]

$$
(\partial / \partial t) \theta_{A}=P_{A}\left(1-\theta_{A}\right)-2 P_{B}\left(1-\theta_{A}\right)^{2}+D_{A}^{0}\left(\partial^{2} / \partial x^{2}\right) \theta_{A}
$$$$
\text { when } \theta_{A}>0 \text { and } \theta_{B}=0 \text {, }
$$

$$
\begin{aligned}
(\partial / \partial t) \theta_{B}= & 2 P_{B}\left(1-\theta_{B}\right)^{2}-P_{A}\left(1-\theta_{B}\right) \\
& +D_{B}^{0}\left(\partial^{2} / \partial x^{2}\right) \theta_{B} \text { when } \theta_{B}>0 \text { and } \theta_{A}=0 .
\end{aligned}
$$

These RDE's can be analyzed using a one-dimensional quasimechanical analogy with conservative "force fields" (cf. Sec. III) [4]. Here, we just consider these equations at equistability, where $\partial / \partial t \theta_{K}=0$, and present the key result from such an analysis [4]. At the single macroscopic point of contact between the $A$ (ads)- and $B$ (ads)-populated regions, the diffusive fluxes of $A$ (ads) and $B$ (ads) (towards this point) are, respectively [4],

$$
\begin{gathered}
J_{A}=\left(D_{A}^{0}\right)^{1 / 2}\left(P_{B}\right)^{1 / 2}\left[P_{A} / P_{B}-4 / 3\right]^{1 / 2} \text { and } \\
J_{B}=\left(D_{B}^{0}\right)^{1 / 2}\left(P_{B}\right)^{1 / 2}\left[\left(P_{A} / P_{B}\right)^{3} / 12-P_{A} / P_{B}+4 / 3\right]^{1 / 2} \\
\text { for } P_{A}=P^{*} .
\end{gathered}
$$

If $h_{B}$ is finite or zero, then spatial correlations persist in the $B$ (ads)-populated region, and the above mean-field description of kinetics is not valid. While one expects that these correlations could be reasonably described even at the level of a pair approximation, appropriate analytic treatment of the sharp chemical wave interface is nontrivial. Nonetheless, it is clear that the above expression for the diffusive flux of $B$ (ads) can be replaced exactly by $J_{B}=0$.

Finally, to obtain explicit values for the equistability point $P^{*}$ from the above flux results, one imposes the condition that $J_{A}=J_{B}$ at the interface between $A$ (ads)- and $B$ (ads)-populated regions (see Ref. [22]). This condition ensures the requisite balance in reactants diffusing to the interface. The following results are obtained in special cases.

(i) $h_{A}=h_{B}=h_{A B}=h \rightarrow \infty$.

(ii) $h_{A}=h_{B}=h \rightarrow \infty$ and $h_{A B} / h \rightarrow 0$.

Setting $D_{A}^{0}=D_{B}^{0}$ in Eq. (6), and requiring that $J_{A}=J_{B}$ yields a cubic equation for $P^{*}=(2 \sqrt{3}-2) /(2 \sqrt{3}-1)$ $\approx 0.594$

(iii) $h_{A}=h_{A B}=h \rightarrow \infty$, and $h_{B} / h \rightarrow 0$.

(iv) $h_{A}=h \rightarrow \infty, h_{B} \rightarrow \infty$ with $h_{B} / h \rightarrow 0$, and $h_{A B} / h \rightarrow 0$.

(v) $h_{A}=h \rightarrow \infty, h_{B}=h_{A B}=0$.

Here we set $J_{B}=0$, and then solving for $J_{A}=0$ yields $P^{*}=4 / 7 \approx 0.571$.

We emphasize that in the last case (v), we are able to obtain an exact result for $P^{*}$ despite the persistence of spatial correlations in the $B(\mathrm{ads})$-populated region. We also note that detailed simulations for this case [13] reveal a nearcrossing feature of $V$ versus $P_{A}$ curves (for various $h$ ), with a near-crossing point at $P_{A}=P_{x}=0.574 \pm 0.003$. This value of $P_{x}$ is consistent with the above exact value of $P^{*}=\frac{4}{7}$. Of course, using Eq. (6), one could also examine the continuous decrease of $P^{*}$ from 0.594 to 0.571 , as one decreases the ratio $D_{B}^{0} / D_{A}^{0}$ from unity towards zero [22]. Finally, note that the substantially higher values of $P^{*}$ for $k=\infty$, compared with $k=1$, should be expected given the higher value of $P^{s+}$.

\section{CONCLUSIONS}

In this study, we have demonstrated that wave propagation and equistability features of the bistable monomer-dimer surface reaction model with $k=1$ in the hydrodynamic regime are significantly influenced by the nontrivial form of chemical diffusion in mixed adlayers. Furthermore, different atomistic prescriptions of adspecies mobility produce distinct behavior in the hydrodynamic limit, and, in particular, distinct equistability points. For finite reaction rates and mixed adlayers, it is only in the special and artificial case where 
rates for different adspecies to hop to vacant sites are equal to each other, and equal to the rate for exchange diffusion, that one recovers the "conventional" diffusion.

It is appropriate to consider generalizations of the above monomer-dimer reaction model. One such natural modification is to introduce nonreactive desorption of the monomer species, with a rate $d>0$. In the LG model, the discontinuous transition is preserved for small $d$, but it disappears as $d$ increases above some critical value $d_{c}[1,23]$, analogous to a critical point in equilibrated systems displaying phase separation. In the hydrodynamic limit, this critical point corresponds to a cusp bifurcation associated with the disappearance of bistability $[4,6]$. The key point of relevance here is that the above prescriptions of chemical diffusion apply to this more general model, and can be used to examine, e.g., chemical wave propagation and equistability for $0 \leqslant d<d_{c}$ $[4,6]$.

Finally, it is appropriate to note that the recent mathematical statistics literature [24] includes some rigorous analyses of behavior of simple reaction models in or near the hydrodynamic limit. However, thus far, such analyses are restricted to situations where chemical diffusion is trivial. In particular, for the simplest case of monomer-dimer model with finite $k$, and $h_{A}=h_{B}=h_{A B} \rightarrow \infty$, the hydrodynamic limit (which we regard as intuitive) is treated rigorously.

\section{ACKNOWLEDGMENTS}

This work was supported by the Division of Chemical Sciences, Office of Basic Energy Sciences, of the U.S. Department of Energy (U.S. DOE). It was performed at Ames Laboratory, which is operated for the U.S. DOE by Iowa State University under Contract No. W-7405-Eng-82.

\section{APPENDIX: LATTICE-GAS MODEL ANALYSIS OF CHEMICAL DIFFUSION}

Consider a mixed adlayer on a square lattice (with lattice constant $a$ ), where species $A$ and $B$ hop to adjacent empty sites at rates $h_{A}$ and $h_{B}$, respectively, and adjacent $A$ and $B$ interchange at rate $h_{A B}$. Let $\left[A_{i j}\right]$ denote the probability that site $(i, j)$ is occupied by $A,\left[A_{i, j} E_{i+1, j}\right]$ the probability that $(i, j)$ is occupied by $A$ and $(i+1, j)$ is empty, etc. Suppose that these probabilities depend only on the column $i$, but not the row $j$ (so the latter label is dropped below). Probability conservation implies that $\left[A_{i}\right]+\left[B_{i}\right]+\left[E_{i}\right]=1,\left[A_{i-1} A_{i}\right]$ $+\left[A_{i-1} B_{i}\right]+\left[A_{i-1} E_{i}\right]=\left[A_{i-1}\right]$, etc. The net diffusive flux of $K=A$ or $B$ from column $i-1$ to column $i$ is denoted $J_{i-1, i}^{K}$, so the diffusive contribution to the rate of change of $\left[K_{i}\right]$ is $\left.(d / d t)\left[K_{i}\right]\right|_{\mathrm{diff}}=J_{i-1, i}^{K}-J_{i, i+1}^{K}$. For these fluxes, one has

$$
\begin{aligned}
J_{i-1, i}^{A}= & h_{A}\left(\left[A_{i-1} E_{i}\right]-\left[E_{i-1} A_{i}\right]\right) \\
& +h_{A B}\left(\left[A_{i-1} B_{i}\right]-\left[B_{i-1} A_{i}\right]\right),
\end{aligned}
$$

and an analogous expression for $J_{i-1, i}^{B}$.

In a mean-field approximation, where one ignores all correlations in the occupancy of adjacent sites, utilizing probability conservation relations, these expressions reduce to

$$
J_{i-1, i}^{A} \approx-h_{A} \Delta\left[A_{i}\right]-\left(h_{A}-h_{A B}\right)\left(\left[A_{i}\right] \Delta\left[B_{i}\right]-\left[B_{i}\right] \Delta\left[A_{i}\right]\right),
$$

where $\Delta\left[K_{i}\right]=\left[K_{i}\right]-\left[K_{i-1}\right]$, and an analogous expression applies for $J_{i-1, i}^{B}$. In the hydrodynamic limit, one makes the replacements $a^{-1} \Delta \rightarrow \nabla$ and $a J_{i-1, i}^{K} \rightarrow J^{K}$. Then, from Eq. (A2), one immediately obtains the approximations $D_{A A}(M F)=D_{A}^{0}-\left(D_{A}^{0}-D_{A B}^{0}\right) \theta_{B} \quad$ and $\quad D_{A B}(M F)=\left(D_{A}^{0}\right.$ $\left.-D_{A B}^{0}\right) \theta_{A}$, where $D_{K}^{0}=a^{2} h_{K}$ and $D_{A B}^{0}=a^{2} h_{A B}$.

Below, we consider some special prescriptions of mobility, and set $D^{0}=a^{2} h$. In a number of these cases, exact analysis is possible. A more detailed discussion of case (c) and case (d) will be presented elsewhere.

(a) Single species adlayer. If only one species $K=A$ or $B$ is present, then $J_{i-1, i}^{K}=-h \Delta\left[K_{i}\right]$ is exactly satisfied, and $\underline{J}^{K}=-D^{0} \nabla \theta_{K}$. This result was noted by Kutner [18].

(b) $h_{A}=h_{B}=h_{A B}=h \rightarrow \infty$. Here, both $J_{i-1, i}^{A}=-h \Delta\left[A_{i}\right]$ and $J_{i-1, i}^{B}=-h \Delta\left[B_{i}\right]$ are exactly satisfied, so one has $D_{J K}$ $=\delta_{J K} D^{0}$, as noted in the text.

(c) $h_{A}=h_{A B}=h \rightarrow \infty ; h_{B} / h \rightarrow 0$. Here $J_{i-1, i}^{A}=-h \Delta\left[A_{i}\right]$ is still exactly satisfied, since the diffusion of the $A$ 's is independent of the $B$ 's, and one has that $D_{A A}=D^{0}$ and $D_{A B}$ $=0$. It is also the case here that the diffusive dynamics of a specific $B$ on the surface is completely independent of the other $B$ 's. (The fast motion of this specific $B$ occurs through interchange with $A$ 's, which are not influenced by the other $B$ 's.) Thus the $B$ evolution is a single-particle problem. This feature, together with the random nature of the $A$ distribution, indicates that $D_{B B}=D^{0} \theta_{A}$ and $D_{B A}=-D^{0} \theta_{B}$ should be given by the MF results. The latter is negative since diffusion of $B$ requires adjacent $A$, and $B$ flows "uphill' in a gradient of $A$. Note that when $\theta=1$, one obtains the result $\underline{J}^{B}=$ $-D^{0} \nabla \theta_{B}$ (where here $\nabla \theta_{A}=-\nabla \theta_{B}$ ) directly and exactly from the above master equations.

(d) $h_{A B}=h \rightarrow \infty ; h_{A} / h$ and $h_{B} / h \rightarrow 0$ [25]. Here, the total coverage $\theta=\theta_{A}+\theta_{B}$ is invariant under diffusion. Thus one has $\underline{J}=\underline{J}_{A}+\underline{J}_{B}=\underline{0}$, and so $D_{A A}+D_{B A}=D_{A B}+D_{B B}=0$. There is also a symmetry condition that if $D_{A A}$ $=D\left(\theta_{A}, \theta_{B}\right)$, then $D_{B B}=D\left(\theta_{B}, \theta_{A}\right)$, so $D$ is determined by the single function $D(x, y)$. [These conditions are satisfied in the MF approximation, where $D_{\mathrm{MF}}(x, y)=D^{0} y$.] If $\theta=\theta_{A}$ $+\theta_{B}$ is constant, then it is also clear that $\underline{J}_{K}=$ $-D_{\text {ant }}(\theta) \nabla \theta_{K}$, for $K=A$ and $B$, where $D_{\text {ant }}$ represents the diffusion coefficient for a single-particle or "ant" moving on the (frozen, possibly nonrandom) set of sites occupied by either $A$ or $B$. Thus one has $D_{\text {ant }}=0$ until $\theta$ reaches the percolation threshold of $A+B$ sites, and then $D_{\text {ant }} \rightarrow D^{0}$, as $\theta \rightarrow 1$. This implies that $D(x, y)+D(y, x)=D_{\text {ant }}(x+y)$, rather than the MF approximation of $D^{0}(x+y)$. One can anticipate that $D(x, y)=p_{y} D_{\text {ant }}(x+y)$, where $p_{y}=y /(x$ $+y)$. Note that when $\theta=1$, one obtains the results $\underline{J}_{K}=$ $-D^{0} \nabla \theta_{K}$, for $K=A$ and $B$, directly and exactly from the above master equations.

(e) $h_{A}=h_{B}=h \rightarrow \infty ; h_{A B} / h \rightarrow 0$.

(f) $h_{A}=h \rightarrow \infty ; h_{B} / h$ and $h_{A B} / h \rightarrow 0$.

In both these cases (e) and (f), nontrivial correlations exist between $A$ and $B$. This results in deviations of diffusion coefficients from the above MF forms, as indicated in Sec. III. 
[1] J. W. Evans, Langmuir 7, 2514 (1991); V. P. Zhdanov and B. Kasemo, Surf. Sci. Rep. 20, 111 (1994); J. W. Evans and M. Sabella, Trends in Statistical Physics (Research Trends, Trivandrum, India, 1994), Vol. 1, p. 107; E. V. Albano, Heterog. Chem. Rev. 3, 389 (1996).

[2] R. Imbihl and G. Ertl, Chem. Rev. 95, 697 (1995).

[3] E. G. Seebauer and C. E. Allen, Prog. Surf. Sci. 49, 265 (1995).

[4] J. W. Evans, J. Chem. Phys. 98, 2463 (1993); 97, 572 (1992).

[5] J. W. Evans and T. R. Ray, Phys. Rev. E 50, 4302 (1994).

[6] M. Tammaro, M. Sabella, and J. W. Evans, J. Chem. Phys. 103, 10277 (1995).

[7] M. Tammaro and J. W. Evans, J. Chem. Phys. 108, 762 (1998).

[8] V. I. Bykov, G. S. Yablonskii, and V. I. Elokhin, Kinet. Katal. 20, 841 (1979); 20, 845 (1979).

[9] R. M. Ziff, E. Gulari, and Y. Barshad, Phys. Rev. Lett. 66, 833 (1986).

[10] M. Dumont, P. Dufour, B. Sente, and R. Daggonier, J. Catal. 122, 95 (1990).

[11] H. P. Kaukonen and R. M. Niemenen, J. Chem. Phys. 91, 4380 (1989); M. Ehsasi, M. Matloch, J. H. Block, K. Christman, F. S. Rys, and W. Hirschwald, ibid. 91, 4949 (1989); I. Jensen, H. Fogedby, and R. Dickman, Phys. Rev. A 41, 3411 (1990); J. Mai, W. von Niessen, and A. Blumen, J. Chem. Phys. 93, 3685 (1990); L. V. Lutsevich, V. I. Elokhin, A. V. Myshlyavtsev, A. G. Uslov, and G. S. Yablonskii, J. Catal. 132, 302 (1991); D. S. Sholl and R. T. Skodje, Surf. Sci. 334, 295 (1995).

[12] In some of the studies in Ref. [11], estimates of $P^{*}$ were corrupted since the system tends to gets trapped in the metastable reactive state just above this transition.

[13] R. H. Goodman, D. S. Graff, L. M. Sander, P. Leroux-Hugon, and E. Clement, Phys. Rev. E 52, 5904 (1995).

[14] R. M. Ziff and B. J. Brosilow, Phys. Rev. A 46, 4630 (1992).

[15] M. Tammaro and J. W. Evans, Surf. Sci. Lett. 395, L207 (1998).

[16] A. S. Mikhailov, Introduction to Synergetics I (Springer, Berlin, 1990).

[17] Note that $\underline{F}$ is not conservative, and its position dependence is only simply determined in the absence of spatial correlations.

[18] R. Kutner, Phys. Lett. 81A, 239 (1981).

[19] See H. Spohn, Large Scale Dynamics of Interacting Particles (Springer, Berlin, 1991); J. Quastel, Commun. Pure Appl. Math. XLV, 623 (1992).

[20] See Table II in Ref. [7]. The entry under "Site approx." labeled "Exact $(R)$ " corresponds to an exact treatment of percolative diffusion in random $B$ adlayers using MF kinetics.

[21] This constitutes the maximum possible value for $P^{s+}$ since the total $A$-adsorption rate, $R_{A}$ (ads) $=P_{A} \theta_{E}$, exceeds that for $B$, $R_{B}$ (ads) $=2 P_{B} \theta_{E E} \geqslant 2 P_{B} \theta_{E}$, when $P_{A}>2 P_{B}$ or $P_{A}>\frac{2}{3}$.

[22] See the review (Ref. [1]) by V. P. Zhdanov and B. Kasemo, Sec. 3.2, p. 125.

[23] B. J. Brosilow and R. M. Ziff, Phys. Rev. A 46, 4534 (1992); E. V. Albano, Appl. Phys. A 54, 1 (1992); T. Tome and R. Dickman, Phys. Rev. E 47, 948 (1993).

[24] R. Durrett and C. Neuhauser, Annals of Probability (Institute of Mathematical Statistics, Hayward, CA, 1994), Vol. 22, p. 289; A. De Masi, A. Pellegrinotti, E. Presutti, and M. E. Vares, ibid. 22, 334 (1994); R. Durrett and G. Swindle, Probability Theory and Related Fields (Springer-Verlag, Berlin, 1994), Vol. 98, p. 489.

[25] For this prescription of mobility (with large $h_{A}$ and $h_{B}$, to ensure randomization of the adlayer, and thus MF kinetics), one finds that $P^{*}(h \rightarrow \infty)=0.381$ in the $A+B_{2}$ reaction model with $k=1$. 